\title{
CONTRIBUIÇÃO DAS DOENÇAS INFECCIOSAS E PARASITÃRIAS NA MORBI-MORTALIDADE DE RIBEIRÃO PRETO, SP (BRASIL)*
}

\author{
José da Rocha Carvalheiro** \\ Clarisse Dulce Gardonyi Carvalheiro** \\ Amábile Rodrigues Xavier ** \\ João Carlos da Costa***
}

\begin{abstract}
RSPUB $9 / 464$
CARVAlheIRo, J. DA R. et al. Contribuição das doenças infecciosas e parasitárias na morbi-mortalidade de Ribeirão Preto, SP (Brasil). Rev. Saúde públ., S. Paulo, 13:203-7, 1979 .

RESUMO: Apresentam-se os resultados da mortalidade e morbidade causadas por doenças infecciosas e parasitáriás (Grupo I da Classificação Internacional de Doenças). Os dados de mortalidade basearam-se em informaçōes da Secretaria de Saúde do Estado de São Paulo; os de morbidade, em estatísticas de egressos hospitalares produzidos pelo Centro de Processamento de Dados Hospitalares, do Departamento de Medicina Social da Faculdade de Medicina de Ribeirão Preto e nos resultados de um levantamento de morbidade por entrevistas domiciliárias. Enquanto as estatísticas de mortalidade exibiram, no ano de 1974 , uma proporçāo de $15,2 \%$ de doenças desse Grupo, em 1975 as estatisticas hospitalares mostraram 3,3\% e as entrevistas domiciliárias $5,2 \%$.
\end{abstract} dade.

Unitermos: Doenças infecciosas. Doenças parasitárias. Mortalidade. Morbi-

I NTRODU S A O

As doenças infecciosas e parasitárias continuam representando um papel importante na morbidade e mortalidade das várias regiões brasileiras, mesmo nas áreas consideradas mais desenvolvidas como as regiōes sudeste e sul do país.

No município de Ribeirão Preto (Estado de São Paulo), apesar da grande freqüência de doenças crônicas e degenerativas, as doenças infecciosas e parasitárias ainda contribuem com razoável parcela da morbi-mortalidade na população.

O presente trabalho tem por objetivo comparar medidas de mortalidade, morbidade hospitalar e por entrevista domiciliária referentes às Doenças Infecçiosas e Parasitárias (Grupo 1 da Classificaçāo Internacional de Doença - CID) ${ }^{3}$ do municipio de Ribeirão Preto, em 1975.

* Apresentado no XIV Congresso da Sociedade Brasileira de Medicina Tropical - João Pessoa, 1978.

* Do Departamento de Medicina Social da Faculdade de Medicina de Ribeirão Preto da USP - "Campus" de Ribeirão Preto - 14100 - Ribeirão Preto, SP - Brasil.

*** Do Departamento de Clínica Médica da Faculdade de Medicina de Ribeirão Preto da USP. 
CARVALHEIRO, J. da $R$, et al. Contribuição das doenças infecciosas e parasitárias na morbimortalidade de Ribeirão Preto, SP (Brasil) Rev. Saúde públ., S. Paulo, 13:203-7. 1979.

\section{MATERIAL E MÉTODOS}

Os dados de mortalidade utilizados foram obtidos da Divisão Regional de Saúde de Ribeirão Preto (DRS-6) da Secretaria de Estado da Saúde de São Paulo, correspondentes ao ano de 1974. Os dados de morbidade foram provenientes das estatísticas de egressos hospitalares produzidas pelo Centro de Processamento de Dados Hospitalares do Departamento de Medicina Social da Faculdade de Medicina de Ribeirão Preto ", em 1975, e dos resultados de um levantamento por entrevistas domiciliárias realizado no mesmo ano.

A mortalidade correspondeu aos óbitos de residentes no municipio de Ribeirão Preto e que aí faleceram, e também aqueles que faleceram em outras localidades da 6" Região Administrativa.

A morbidade hospitalar correspondeu às internaçōes de residentes ocorridas em todos os hospitais de Ribeirão Preto, enquanto que a morbidade por entrevistas domiciliárias correspondeu a uma amostra representativa da população da cidade de Ribeirão Preto?. Essa amostra foi retirada de um painel de 5.200 domicilios, dos quais 380 eram visitados semanalmente.

\section{RESULTADOS E DISCUSSĀO}

Os dados de mortalidade referem-se ao ano de 1974, uma vez que não se dispunham dos dados referentes a 1975 . Entretanto, acredita-se que não tenha havido grandes variaçōes nos anos próximos a 1975; assim, em 1972, por exemplo, o coeficiente geral de mortalidade foi de 7,2 por mil habitantes e o coeficiente especifico de mortalidade para o Grupo I da CID (Revisão de 1965) ${ }^{ \pm}$ foi de 0,9 por mil habitantes. A mortalidade proporcional do Grupo I, para o mesmo ano, foi de $12,1 \%$. Em 1974, ano de referência utilizado, obteve-se para as mesmas medidas acima referidas, 7,1 por mil habitantes, 1,1 por mil habitantes e $15,2 \%$ respectivamente.
A proporção de morbidade hospitalar para o Grupo de Doenças Infecciosas e Parasitárias foi de $3,3 \%$ valor que se assemelha ao de países desenvolvidos como o Canadá (3,4\% em 1972) e Estados Unidos $(2,9 \%$ em 1974); já alguns países latino-americanos mostram valores maiores; assim, 8,9\% para a Costa Rica, em 1974. e $7,9 \%$ para o Paraguai, em 1975 .

Deve-se lembrar, entretanto, que essas diferentes proporções são também afetadas por numerosos fatores relacionados alguns com a qualidade do diagnóstico e com a variação no tipo de informação produzida pelos hospitais. Sem entrar no mérito do método empregado no fornecimento e coleta de informações, não se pode afastar a hipótese de que as diferenças sejam devidas a meros artefatos.

Esta observação, aliás, vale para dados de morbidade como de mortalidade, merecendo por parte de diversos autores uma atenção especial no sentido de afastar distorções. A Investigação Interamericana de Mortalidade na Infâncias, por exemplo, assinala que o registro de óbitos neonatais é tão incompleto que não permite o conhecimento suficiente de mortalidade nesse grupo etário; entre as recomendações feitas como resultado da investigação, os autores chamam a atenção para o fato de que devem ser tomadas medidas imediatas para melhorar a integridade e qualidade das estatísticas vitais, estatísticas hospitalares e do atestado de óbito. Quanto à morbidade referida pela população, ela foi levantada em 25 das semanas de 1975 . O número de familias entrevistadas, respectivos membros, episódios de morbidade referidos (em particular, os dos Grupo 1 da CID), estão apresentados na Tabela e os coeficientes de morbidade na Fig. Nesta observa-se que o coeficiente geral de morbidade referida exibe uma tendência decrescente, mantendo-se a morbidade específica do Grupo I da CID, o que conduz a um ligeiro aumento da morbidade proporcional. 
CARVAlHEIro. J. da R. et al. Contribuição das doenças infecciosas e parasitárias na morbimortalidade de Ribeirão Preto, SP (Brasil) Rev. Saúde públ., S. Paulo, 13:203-7. 1979.

\section{T A B E L A}

Famillas entrevistadas, número de membros e morbidades referidas. segundo a semani epidemiológica. Ribeirão Preto. 1975.

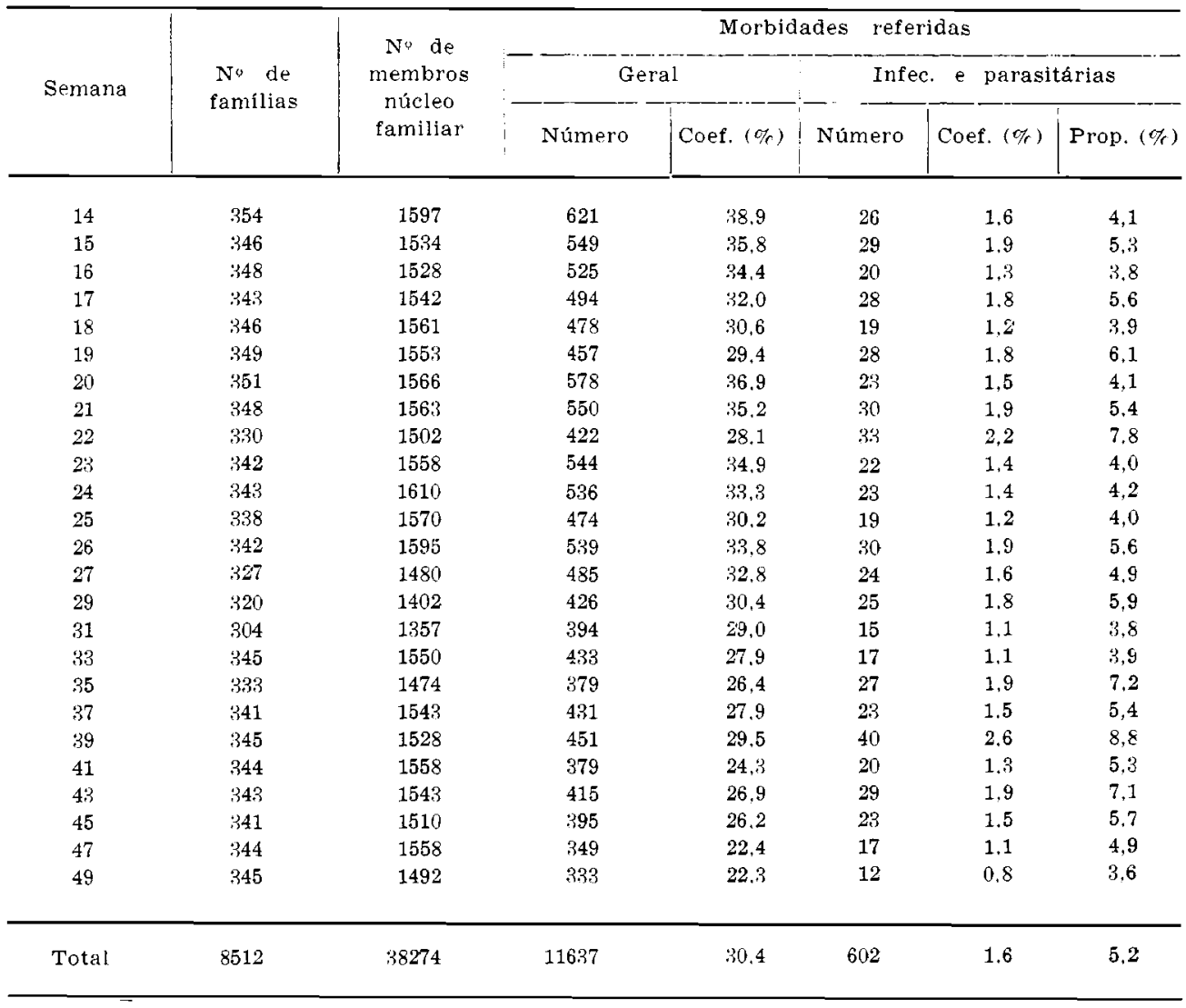

Considerando os resultados globalmente (Tabela), tem-se um coeficiente de morbidade geral de 30,4 episódios de doença por cem pessoas, o que não é exagerado se levarmos em conta que ai estão incluídos todos os tipos de doenças, desde resfriados triviais e "dores de cabeça" até patologias graves. Isolando-se apenas as doenças infecciosas e parasitárias, observamos que elas participaram com $5,2 \%$ da morbidade referida, isto é, com 1,6 episódios de doença por cem pessoas.

Vale a pena ainda analisar que peso representam as várias doenças do Grupo I quanto a mortalidade e morbidade. Assim, verifica-se que 58,9\% dos óbitos de 1974 devem-se à doença de Chagas, $16,2 \%$ à doença diarréica, $9,4 \%$ à tuberculose e $8,3 \%$ às infecções meningocócicas. Já na morbidade hospitalar o quadro se altera: a doença diarréica corresponde a $39,3 \%$ do Grupo I, a doença de Chagas contribui com apenas $7,8 \%$ e as infecções meningocócicas com $5,3 \%$.

Merece ainda reparo que o problema das doenças infecciosas e parasitárias é bastante complexo visto que sua incidencia é fortemente influenciada pelas condições ambientais. Outra consideração a ser feita é quanto ao grau de importância das doẹn- 
CARVAlHEIRO, J. da R. et al. Contribuição das doenças infecciosas e parasitárias na morbimortalidade de Ribeirão Preto, SP (Brasil) Rev. Saúde públ., S. Paulo, 13:203-7, 1979.

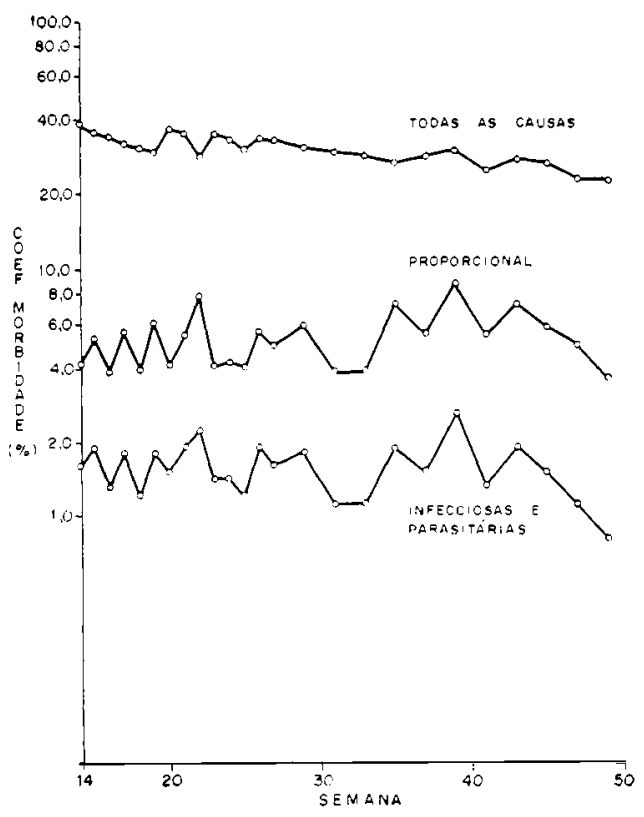

Morbidade referida nas entrevistas domiciliárıas, segundo a semana epidemiológica - Ribeirão Preto. 1975.

ças infecciosas e parasitárias a nivel do sistema de ensino médico no nosso país, uma vez que grande parte dele ainda se realiza dentro dos hospitais de ensino. Em 1961, White e col.' já referiam, em seu modelo ecológico de assistência médica, a distorção representada pelo ensino médico exclusivamente a nível de hospital universitário. De cada mil pessoas, 750 referiam, num mês, alguns episódios de morbidade, 250 eram atendidas por médico, 9 chegavam a hospitalizar-se e apenas uma era referida ao hospital de ensino. Evidentemente, este modelo não tem aplicação universal. Conforme já acentuado por Carvalheiro ${ }^{1}$, o processo de assistência médica é único para cada época e lugar e está na dependência de inúmeros fatores determinados historicamente. Não obtante isso, seria interessante imaginar como funciona referido modelo, aplicado em nosso contexto. Inicialmente, é de se prever que as proporçōes citadas por White e coli. va- riem com o nivel de renda, o que de fato se verifica lançando sobre a compreensão do fenômeno luzes muito mais da esfera social do que da biológica. Uma interpretação do processo do ensino médico na linha de White e col. nos levaria a dois planos. Naquele em que apenas os casos mais graves ou patologias raras chegariam ao hospital universitário, deixando de dar ao estudante, formado exclusivamente a esse nivel, uma visão global dos problemas de saúde e, portanto, de treiná-lo para enfrentá-los. Num outro plano, poderiamos imaginar as distorções ainda mais sérias determinadas pelo distinto acesso à assistência médica, pelos diversos estratos de renda.

No campo das doenças infecciosas e parasitárias, um exemplo específico é o da doença de Chagas, que apareceria em Ribeirão Preto, ainda como importante causa de óbito (corresponde a 58,9\% dos óbitos do Grupo I), apesar de já estar totalmente controlada na região, há muitos anos, o que se reflete na pequena parcela de internações $(7,8 \%$ da morbidade hospitalar por doenças infecciosas e parasitárias) e na ausência de referências a doença de Chagas na comunidade.

Pelas razões sugeridas, o fenômeno não é de interpretação tão simples. Os próprios dados de que dispomos deveriam ser cuidadosamente avaliados, em busca de maior consistência. Não obstante é possivel que algumas das impressões colhidas nos informes oficiais de serviços de saúde do chamado sistema formal devessem ser revistas. Como, por exemplo, a que afirma estarem as doenças infecciosas e parasitárias praticamente controladas na região sudeste. Os reflexos de semelhantes opiniōes são fáceis de prever, não apenas quanto ao sistema de prestação de serviços, mas principalmente ao nivel do sistema de ensino médico. 
CARVAlheiro, J. da $R$. et al. Contribuição das doenças infecciosas e parasitárias na morbimortalidade de Ribeirão Preto, SP (Brasil) Rev. Saúde públ., S. Paulo, 13:203-7, 1979.

\section{CONCLUSOES}

1. As doenças infecciosas e parasitárias ainda permanecem como causa importante da morbi-mortalidade de Ribeirão Preto.

2. A mortalidade geral de residentes, em 1974 , foi de 7,1 por mil habitantes, o coeficiente específico de mortalidade para o Grupo 1 da CID foi de 1,1 por mil habitantes e a mortalidade proporcional $15,2 \%$.
3. A morbidade hospitalar, em 1975, foi de $3,3 \%$ para as doenças infecciosas e parasitárias, sendo $39,3 \%$ delas correspondentes à doença diarréica, $7,8 \%$ à doença de Chagas e $5,3 \%$ às infecçōes meningocócicas.

4. A morbidade por entrevistas domiciliárias foi de 30,4 episódios de doença por 100 pessoas, sendo $5,2 \%$ correspondentes às doenças infecciosas e parasitárias (1,6 episódios por 100 pessoas).

\section{RSPUB9/464}

CARVALHEIRO, J. DA R. et al. IThe contribution of infectious and parasitic diseases to the morbidity-mortality rates of Ribeirão Preto, $S P$ (Brazil))]. Rev. Saúde pábl., S. Paulo, 13:203-7, 1979.

ABSTRACT: Mortality and morbidity caused by infectious and parasitic diseases (Group I of the International Classification of Diseases) were studied. Mortality statistics were obtained from the S. Paulo State Health Department; morbidity, from hospital statistics furnished by the Hospital Data Processing Center of the Department of Social Medicine of the School of Medicine of Ribeirão Preto and from information obtained by a home survey. In 1974, mortality statistics showed a $15.2 \%$ proportion of infectious and parasitic diseases; however, in 1975, the hospital statistics for morbidity were $3.3 \%$ and $5.2 \%$ in the home survey.

Uniterms: Communicable diseases. Parasitic diseases. Mortality. Morbidity.

\section{REFERENCIAS RIBLIOGRAFICAS}

1. CaRValHeiro, J. R. Epidemiological research and health manpower development. [apresentado ao 8th International Scientific Meeting - International Epidemiological Association, San Juan, Puerto Rico, 1977].

2. CARVALHEIRo, J. R. Levantamento de condiçōes de saúde por entrevistas domiciliarias. Ribeirão Preto, 1975. [Tese de Livre-Docência - Faculdade de Medicina de Ribeirão Preto da USPJ.

3. ORGANIZAÇO PANAMERICANA DA SAƯDE. Manual da classificação estatis. tica internacional de doencas, lesos $e$ causas de bbitos; revisão 1965. Washington, D.C., 1969, v. 1 .

4. ORganizacion PANAMERICANA DE IA SALUD, Las condiciones de salud en las Americas: 1973-1976. Washington.

D, C., 1978. (Publ. cient., 364).

5. PUFFER, R. R. \& SERRANO, C. V. Caracteristicas de la mortalidad en la niñez. Washington, D.C., Organizacion Panamericana de la Salud, 1973. (OPASPubl. cient., 262).

6. UNIVERSIDADE DE SAO PAULO. Faculdade de Medicina. Departamento de Medicina Social. Levantamento continuo de morbidade $e$ mortalidade hospitalar. Ribeirão Preto, 1975.

7. WHITE, K. L. et al. The ecology of medical care. New Engl. J, Med., 265:885-92, 1961.

Recebido para publicacto em 22/03/1979 Aprovado para publicafdo em 19/06/1979 